J. Korean Math. Soc. 50 (2013), No. 3, pp. 465-477

http://dx.doi.org/10.4134/JKMS.2013.50.3.465

\title{
TIME SCALES INTEGRAL INEQUALITIES FOR SUPERQUADRATIC FUNCTIONS
}

\author{
Josipa Barić, Rabia Bibi, Martin Bohner, And Josip PeČarić
}

ABSTRACT. In this paper, two different methods of proving Jensen's inequality on time scales for superquadratic functions are demonstrated. Some refinements of classical inequalities on time scales are obtained using properties of superquadratic functions and some known results for isotonic linear functionals.

\section{Introduction}

\subsection{On time scale calculus}

The theory of time scales was introduced by Stefan Hilger in his Ph.D. thesis [12] in 1988 as a unification of the theory of difference equations with that of differential equations, unifying integral and differential calculus with the calculus of finite differences, extending to cases "in between", and offering a formalism for studying hybrid discrete-continuous dynamic systems. It has applications in any field that requires simultaneous modelling of discrete and continuous data. Now, we briefly introduce the time scales calculus and refer to $[13,14]$ and the monograph [10] for further details.

By a time scale $\mathbb{T}$ we mean any nonempty closed subset of $\mathbb{R}$. The two most popular examples of time scales are the real numbers $\mathbb{R}$ and the integers $\mathbb{Z}$. Since the time scale $\mathbb{T}$ may or may not be connected, we need the concept of jump operators.

For $t \in \mathbb{T}$, we define the forward jump operator $\sigma: \mathbb{T} \rightarrow \mathbb{T}$ by

$$
\sigma(t)=\inf \{s \in \mathbb{T}: s>t\}
$$

and the backward jump operator by

$$
\rho(t)=\sup \{s \in \mathbb{T}: s<t\} .
$$

In this definition, the convention is $\inf \emptyset=\sup \mathbb{T}$ and $\sup \emptyset=\inf \mathbb{T}$.

Received November 24, 2011.

2010 Mathematics Subject Classification. Primary 26D15, 26D20, 26D99, 34 N05.

Key words and phrases. time scales, superquadratic functions, Jensen's inequality, Hölder's inequality, Minkowski's inequality, Jessen-Mercer's inequality, Slater's inequality, Hermite-Hadamard's inequality. 
If $\sigma(t)>t$, then we say that $t$ is right-scattered, and if $\rho(t)<t$, then we say that $t$ is left-scattered. Points that are right-scattered and left-scattered at the same time are called isolated. Also, if $\sigma(t)=t$, then $t$ is said to be right-dense, and if $\rho(t)=t$, then $t$ is said to be left-dense. Points that are simultaneously right-dense and left-dense are called dense. If $\mathbb{T}$ has a left-scattered maximum $M$, then we define $\mathbb{T}^{\kappa}=\mathbb{T} \backslash\{M\}$; otherwise $\mathbb{T}^{\kappa}=\mathbb{T}$.

The mapping $\mu: \mathbb{T} \rightarrow[0, \infty)$ defined by

$$
\mu(t)=\sigma(t)-t
$$

is called the forward graininess function.

In the following considerations, $\mathbb{T}$ will denote a time scale, $I_{\mathbb{T}}=I \cap \mathbb{T}$ will denote a time scale interval (for any open or closed interval $I$ in $\mathbb{R}$ ), and $[0, \infty)_{\mathbb{T}}$ will be used for the time scale interval $[0, \infty) \cap \mathbb{T}$.

Definition 1.1. Assume $f: \mathbb{T} \rightarrow \mathbb{R}$ is a function and let $t \in \mathbb{T}^{\kappa}$. Then we define $f^{\Delta}(t)$ to be the number (provided it exists) with the property that given any $\varepsilon>0$, there is a neighborhood $U$ of $t$ such that

$$
\left|(f(\sigma(t))-f(s))-f^{\Delta}(t)(\sigma(t)-s)\right| \leq \varepsilon|\sigma(t)-s| \quad \text { for all } \quad s \in U_{\mathbb{T}} .
$$

We call $f^{\Delta}(t)$ the delta derivative of $f$ at $t$. We say that $f$ is delta differentiable on $\mathbb{T}^{\kappa}$ provided $f^{\Delta}(t)$ exists for all $t \in \mathbb{T}^{\kappa}$.

For all $t \in \mathbb{T}^{\kappa}$, we have the following properties.

(i) If $f$ is delta differentiable at $t$, then $f$ is continuous at $t$.

(ii) If $f$ is continuous at $t$ and $t$ is right-scattered, then $f$ is delta differentiable at $t$ with $f^{\Delta}(t)=\frac{f(\sigma(t))-f(t)}{\mu(t)}$.

(iii) If $t$ is right-dense, then $f$ is delta differentiable at $t$ if and only if $\lim _{s \rightarrow t} \frac{f(t)-f(s)}{t-s}$ exists as a finite number. In this case, $f^{\Delta}(t)=\lim _{s \rightarrow t} \frac{f(t)-f(s)}{t-s}$.

(iv) If $f$ is delta differentiable at $t$, then $f(\sigma(t))=f(t)+\mu(t) f^{\Delta}(t)$.

Definition 1.2. A function $f: \mathbb{T} \rightarrow \mathbb{R}$ is called $r d$-continuous if it is continuous at all right-dense points in $\mathbb{T}$ and its left-sided limits are finite at all left-dense points in $\mathbb{T}$. We denote by $\mathrm{C}_{\mathrm{rd}}$ the set of all $\mathrm{rd}$-continuous functions. We say that $f$ is rd-continuously delta differentiable (and write $f \in \mathrm{C}_{\mathrm{rd}}^{1}$ ) if $f^{\Delta}(t)$ exists for all $t \in \mathbb{T}^{\kappa}$ and $f^{\Delta} \in \mathrm{C}_{\mathrm{rd}}$.

The set of all continuous functions on $\mathbb{T}$ contains $\mathrm{C}_{\mathrm{rd}}$.

Definition 1.3. A function $F: \mathbb{T} \rightarrow \mathbb{R}$ is called a delta antiderivative of $f: \mathbb{T} \rightarrow \mathbb{R}$ if $F^{\Delta}(t)=f(t)$ for all $t \in \mathbb{T}^{\kappa}$. Then we define the delta integral by

$$
\int_{a}^{t} f(s) \Delta s=F(t)-F(a)
$$

The importance of rd-continuous function is revealed by the following result.

Theorem 1.4 (See [10, Theorem 1.74]). Every rd-continuous function has a delta antiderivative. 
Now we give some properties of the delta integral.

Theorem 1.5 (See [10, Theorem 1.77]). If $a, b, c \in \mathbb{T}, \alpha \in \mathbb{R}$ and $f, g \in \mathrm{C}_{\mathrm{rd}}$, then

(i) $\int_{a}^{b}(f(t)+g(t)) \Delta t=\int_{a}^{b} f(t) \Delta t+\int_{a}^{b} g(t) \Delta t$;

(ii) $\int_{a}^{b} \alpha f(t) \Delta t=\alpha \int_{a}^{b} f(t) \Delta t$;

(iii) $\int_{a}^{b} f(t) \Delta t=-\int_{b}^{a} f(t) \Delta t$;

(iv) $\int_{a}^{b} f(t) \Delta t=\int_{a}^{c} f(t) \Delta t+\int_{c}^{b} f(t) \Delta t$;

(v) $\int_{a}^{a} f(t) \Delta t=0$;

(vi) if $f(t) \geq 0$ for all $t$, then $\int_{a}^{b} f(t) \Delta t \geq 0$.

\subsection{On superquadratic functions}

The concept of superquadratic functions in one variable, as a generalization of the class of convex functions, was recently introduced by S. Abramovich, G. Jameson and G. Sinnamon in [3,4]. Here we quote some definitions and theorems that we use in this paper. More examples and properties of superquadratic functions can be found in $[1,7,9]$ and its references.

Definition 1.6. A function $\varphi:[0, \infty) \rightarrow \mathbb{R}$ is called superquadratic if there exists a function $C:[0, \infty) \rightarrow \mathbb{R}$ such that

$$
\varphi(y)-\varphi(x)-\varphi(|y-x|) \geq C(x)(y-x) \text { for all } x, y \geq 0 .
$$

We say that $\varphi$ is subquadratic if $-\varphi$ is superquadratic.

For example, the function $\varphi(x)=x^{p}$ is superquadratic for $p \geq 2$ and subquadratic for $p \in(0,2]$.

The following lemma shows essentially that positive superquadratic functions are also convex functions.

Lemma 1.7. Let $\varphi$ be a superquadratic function with $C$ as in Definition 1.6. Then

(i) $\varphi(0) \leq 0$

(ii) if $\varphi(0)=\varphi^{\prime}(0)=0$, then $C(x)=\varphi^{\prime}(x)$ whenever $\varphi$ is differentiable at $x>0$;

(iii) if $\varphi \geq 0$, then $\varphi$ is convex and $\varphi(0)=\varphi^{\prime}(0)=0$.

\section{Jensen's inequality}

Jensen's inequality is of great interest in the theory of differential and difference equations as well as other areas of mathematics. The original Jensen inequality can be stated as follows (see also [16]).

Theorem 2.1 (See $\left[15\right.$, Formula $\left.\left.\left(5^{\prime}\right)\right]\right)$. If $g \in \mathrm{C}([a, b],(c, d))$ and $f \in \mathrm{C}((c, d), \mathbb{R})$ is convex, then

$$
f\left(\frac{\int_{a}^{b} g(s) \mathrm{d} s}{b-a}\right) \leq \frac{\int_{a}^{b} f(g(s)) \mathrm{d} s}{b-a} .
$$


Jensen's inequality for a superquadratic function is given in the following theorem.

Theorem 2.2 (See [9, Theorem 1.4.1]). The inequality

$$
f\left(\int g \mathrm{~d} \mu\right) \leq \int\left(f(g(s))-f\left(\left|g(s)-\int g \mathrm{~d} \mu\right|\right)\right) \mathrm{d} \mu(s)
$$

holds for all probability measures $\mu$ and all nonnegative $\mu$-integrable functions $g$, if and only if $f$ is superquadratic.

S. Banić and S. Varošanec proved in [8] the Jensen inequality for isotonic linear functionals and superquadratic functions. First we introduce isotonic linear functionals.

Let $E$ be a nonempty set and $L$ be a linear class of real-valued functions $f: E \rightarrow \mathbb{R}$ having the properties:

$\left(\mathrm{L}_{1}\right)$ If $f, g \in L$ and $\alpha, \beta \in \mathbb{R}$, then $(\alpha f+\beta g) \in L$.

$\left(\mathrm{L}_{2}\right)$ If $f(t)=1$ for all $t \in E$, then $f \in L$.

An isotonic linear functional is a functional $A: L \rightarrow \mathbb{R}$ having the properties:

$\left(\mathrm{A}_{1}\right)$ If $f, g \in L$ and $\alpha, \beta \in \mathbb{R}$, then $A(\alpha f+\beta g)=\alpha A(f)+\beta A(g)$.

$\left(\mathrm{A}_{2}\right)$ If $f \in L$ and $f(t) \geq 0$ for all $t \in E$, then $A(f) \geq 0$.

Now, we quote the following result of Banić and Varošanec.

Theorem 2.3 (See [8, Theorem 10]). Let L satisfy conditions $\left(\mathrm{L}_{1}\right),\left(\mathrm{L}_{2}\right)$ and $A$ satisfy conditions $\left(\mathrm{A}_{1}\right)$ and $\left(\mathrm{A}_{2}\right)$ on a nonempty set $E$. Suppose that $k \in L$ with $k \geq 0$ and $A(k)>0$ and that $\varphi:[0, \infty) \rightarrow \mathbb{R}$ is a continuous superquadratic function. Then for all nonnegative $f \in L$ such that

$$
k f, k \varphi(f), k \varphi\left(\left|f-\frac{A(k f)}{A(k)} \cdot 1\right|\right) \in L,
$$

we have

$$
\varphi\left(\frac{A(k f)}{A(k)}\right) \leq \frac{A(k \varphi(f))-A\left(k \varphi\left(\left|f-\frac{A(k f)}{A(k)} \cdot 1\right|\right)\right)}{A(k)} .
$$

If $\varphi$ is a subquadratic function, then a reversed inequality holds.

The Jensen inequality on time scales via the $\Delta$-integral has been recently obtained in [5] by Agarwal, Bohner and Peterson.

Theorem 2.4 (See [10, Theorem 6.17]). Let $a, b \in \mathbb{T}$ and $c, d \in \mathbb{R}$. Suppose $f$ : $[a, b]_{\mathbb{T}}^{\kappa} \rightarrow(c, d)$ is rd-continuous and $F:(c, d) \rightarrow \mathbb{R}$ is convex (resp., concave). Then

$$
F\left(\frac{\int_{a}^{b} f(t) \Delta t}{b-a}\right) \leq \frac{\int_{a}^{b} F(f(t)) \Delta t}{b-a}
$$

(resp., the reversed inequality is valid). Moreover, if $F$ is strictly convex or strictly concave, then equality in (2) holds if and only if $f$ is constant. 
In this section we will demonstrate how Jensen's inequality on time scales for superquadratic functions can be proved by two completely different approaches: The first approach uses the methods and techniques of time scales calculus and the second one follows from Theorem 2.3. According to the conclusion that comes out from the second way of proving Jensen's inequality, in the rest of this paper, some new inequalities with delta-integrals will be obtained.

Now we present Jensen's inequality on time scales for superquadratic functions.

Theorem 2.5. Let $a, b \in \mathbb{T}$. Suppose $f:[a, b]_{\mathbb{T}}^{\kappa} \rightarrow[0, \infty)$ is rd-continuous and $\varphi:[0, \infty) \rightarrow \mathbb{R}$ is continuous and superquadratic. Then

$$
\varphi\left(\frac{\int_{a}^{b} f(t) \Delta t}{b-a}\right) \leq \frac{1}{b-a} \int_{a}^{b}\left[\varphi(f(s))-\varphi\left(\left|f(s)-\frac{\int_{a}^{b} f(t) \Delta t}{b-a}\right|\right)\right] \Delta s .
$$

First Proof of Theorem 2.5. Let $\varphi:[0, \infty) \rightarrow \mathbb{R}$ be a superquadratic function and let $x_{0} \in[0, \infty)$. According to $(1)$, there is a constant $C\left(x_{0}\right)$ such that

$$
\varphi(y) \geq \varphi\left(x_{0}\right)+C\left(x_{0}\right)\left(y-x_{0}\right)+\varphi\left(\left|y-x_{0}\right|\right) .
$$

Since $f$ is rd-continuous,

$$
x_{0}=\frac{\int_{a}^{b} f(t) \Delta t}{b-a}
$$

is well defined. The function $\varphi \circ f$ is also rd-continuous, so we may apply (4) with $y=f(s)$ and (5) to obtain

$$
\begin{aligned}
\varphi(f(s)) \geq & \varphi\left(\frac{\int_{a}^{b} f(t) \Delta t}{b-a}\right)+C\left(x_{0}\right)\left(f(s)-\frac{\int_{a}^{b} f(t) \Delta t}{b-a}\right) \\
& +\varphi\left(\left|f(s)-\frac{\int_{a}^{b} f(t) \Delta t}{b-a}\right|\right) .
\end{aligned}
$$

Integrating (6) from $a$ to $b$, we get

$$
\begin{aligned}
& \int_{a}^{b}\left[\varphi(f(s))-\varphi\left(\left|f(s)-\frac{\int_{a}^{b} f(t) \Delta t}{b-a}\right|\right)\right] \Delta s-(b-a) \varphi\left(\frac{\int_{a}^{b} f(t) \Delta t}{b-a}\right) \\
= & \int_{a}^{b} \varphi(f(s)) \Delta s-\int_{a}^{b} \varphi\left(\left|f(s)-\frac{\int_{a}^{b} f(t) \Delta t}{b-a}\right|\right) \Delta s-\int_{a}^{b} \varphi\left(\frac{\int_{a}^{b} f(t) \Delta t}{b-a}\right) \Delta s \\
\geq & C\left(x_{0}\right) \int_{a}^{b}\left[f(s)-\frac{\int_{a}^{b} f(t) \Delta t}{b-a}\right] \Delta s \\
= & C\left(x_{0}\right)\left[\int_{a}^{b} f(s) \Delta s-(b-a) \cdot x_{0}\right] \\
= & 0,
\end{aligned}
$$


from which (3) follows.

The methods that will be used in the second proof of Theorem 2.5 are based on the following consequence of Theorem 1.5.

Theorem 2.6 (See [6, Theorem 3.2]). Let $\mathbb{T}$ be a time scale. For $a, b \in \mathbb{T}$ with $a<b$, let

$$
E=[a, b) \cap \mathbb{T} \quad \text { and } \quad L=\mathrm{C}_{\mathrm{rd}}([a, b), \mathbb{R}) .
$$

Then $\left(\mathrm{L}_{1}\right)$ and $\left(\mathrm{L}_{2}\right)$ are satisfied. Moreover, let

$$
A(f)=\int_{a}^{b} f(t) \Delta t
$$

where the integral is the delta integral. Then $\left(A_{1}\right)$ and $\left(A_{2}\right)$ are satisfied.

Second Proof of Theorem 2.5. Substituting $A$ from Theorem 2.6 into Theorem 2.3 and using $k(t)=1$ for all $t \in[a, b]_{\mathbb{T}}^{\kappa}$, we get inequality $(3)$.

Remark 2.7. In the case when $\varphi$ is a nonnegative superquadratic function and therefore (by Lemma 1.7) a convex one too, the result of Theorem 2.5 refines the result given in Theorem 2.4 .

In $[2,8]$, some classical inequalities are proved for isotonic linear functionals and superquadratic functions. In the rest of this paper, using Theorem 2.6 and the results from $[2,8]$, some new inequalities for time scales integrals and superquadratic functions will be obtained. Obviously, according to Theorem 2.6 , it is not necessary to prove those new inequalities following the methods and techniques of time scales calculus as they can be obtained easily from the well-known inequalities for isotonic linear functionals.

\section{Hölder's inequality}

Let us recall the following refinement of the functional Hölder inequality.

Theorem 3.1 (See [8, Theorem 13]). Let L satisfy conditions $\left(\mathrm{L}_{1}\right),\left(\mathrm{L}_{2}\right)$ and let $A$ satisfy conditions $\left(\mathrm{A}_{1}\right),\left(\mathrm{A}_{2}\right)$ on a nonempty set $E$. Let $p \neq 1$ and define $q$ by $\frac{1}{p}+\frac{1}{q}=1$. If $p \geq 2$, then for all nonnegative functions $g, h \in L$ such that $g h, g^{p}, h^{q},\left|g-h^{q-1} \frac{A(g h)}{A\left(h^{q}\right)}\right|^{p} \in L$, and $A\left(h^{q}\right)>0$, the inequality

$$
A(g h) \leq\left[A\left(g^{p}\right)-A\left(\left|g-h^{q-1} \frac{A(g h)}{A\left(h^{q}\right)}\right|^{p}\right)\right]^{\frac{1}{p}} A^{\frac{1}{q}}\left(h^{q}\right)
$$

holds. In the case $0<p<2$, the inequality in (7) is reversed.

Now Hölder's inequality on time scales (see [5] and [10, Theorem 6.13]) can be refined as follows. 
Theorem 3.2. Let $a, b \in \mathbb{T}$. Let $p \neq 1$ and define $q$ by $\frac{1}{p}+\frac{1}{q}=1$. If $p \geq 2$, then, for all rd-continuous functions $g, h:[a, b]_{\mathbb{T}} \rightarrow[0, \infty)$ with $\int_{a}^{b} h^{q}(t) \Delta t>0$, the inequality

(8) $\int_{a}^{b}(g h)(t) \Delta t$

$$
\leq\left[\int_{a}^{b} g^{p}(t) \Delta t-\int_{a}^{b}\left(\left|g(s)-h^{q-1}(s) \frac{\int_{a}^{b}(g h)(t) \Delta t}{\int_{a}^{b} h^{q}(t) \Delta t}\right|^{p}\right) \Delta s\right]^{\frac{1}{p}}\left(\int_{a}^{b} h^{q}(t) \Delta t\right)^{\frac{1}{q}}
$$

holds. In the case $0<p<2$, the inequality in (8) is reversed.

Proof. The inequality (8) follows from Theorem 3.1 and Theorem 2.6.

Remark 3.3. Since the delta integral is an isotonic linear functional, we have

$$
\int_{a}^{b} h^{q}(t) \Delta t \geq 0 \quad \text { and } \quad \int_{a}^{b}\left(\left|g(s)-h^{q-1}(s) \frac{\int_{a}^{b}(g h)(t) \Delta t}{\int_{a}^{b} h^{q}(t) \Delta t}\right|^{p}\right) \Delta s \geq 0,
$$

so the inequality (8) represents a refinement of the classical Hölder inequality on time scales for nonnegative functions $g$ and $h$.

Taking $p=q=2$ in Theorem 3.2 gives the following special case of the above Hölder inequality that we can name the refinement of the Cauchy-Schwarz inequality on time scales.

Theorem 3.4. Let $a, b \in \mathbb{T}$. For $r d$-continuous functions $g, h:[a, b]_{\mathbb{T}} \rightarrow[0, \infty)$ with $\int_{a}^{b} h^{2}(t) \Delta t>0$, the inequality

(9) $\int_{a}^{b}(g h)(t) \Delta t$

$$
\leq\left[\int_{a}^{b} g^{2}(t) \Delta t-\int_{a}^{b}\left(\left|g(s)-h(s) \frac{\int_{a}^{b}(g h)(t) \Delta t}{\int_{a}^{b} h^{2}(t) \Delta t}\right|^{2}\right) \Delta s\right]^{\frac{1}{2}}\left(\int_{a}^{b} h^{2}(t) \Delta t\right)^{\frac{1}{2}}
$$

holds.

\section{Minkowski's inequality}

First, we quote the following functional Minkowski inequality for superquadratic functions.

Theorem 4.1 (See [8, Theorem 14]). Let L satisfy conditions $\left(\mathrm{L}_{1}\right),\left(\mathrm{L}_{2}\right)$ and let $A$ satisfy conditions $\left(\mathrm{A}_{1}\right),\left(\mathrm{A}_{2}\right)$ on a nonempty set $E$. If $p \geq 2$ and $A(g+h)^{p}>$ 0 , then for all nonnegative functions $g, h$ on $E$ such that $(g+h)^{p}, g^{p}, h^{p} \in L$, the inequality

$$
A^{\frac{1}{p}}\left((g+h)^{p}\right) \leq\left(A\left(g^{p}\right)-A\left(\left|g-(g+h) \frac{A\left(g(g+h)^{p-1}\right)}{A(g+h)^{p}}\right|^{p}\right)\right)^{\frac{1}{p}}
$$




$$
+\left(A\left(h^{p}\right)-A\left(\left|h-(g+h) \frac{A\left(h(g+h)^{p-1}\right)}{A(g+h)^{p}}\right|^{p}\right)\right)^{\frac{1}{p}}
$$

holds.

Now, Minkowski's inequality on time scales (see [5] and [10, Theorem 6.16]) can be refined as follows.

Theorem 4.2. Let $a, b \in \mathbb{T}$ and $p \geq 2$. For rd-continuous functions $g, h$ : $[a, b]_{\mathbb{T}} \rightarrow[0, \infty)$ with $\int_{a}^{b}(g(s)+h(s))^{p} \Delta s>0$, the inequality

$$
\begin{aligned}
& \left(\int_{a}^{b}(g(t)+h(t))^{p} \Delta t\right)^{\frac{1}{p}} \\
\leq & \left(\int_{a}^{b} g^{p}(t) \Delta t-\int_{a}^{b}\left|g(t)-(g(t)+h(t)) \frac{\int_{a}^{b} g(s)(g(s)+h(s))^{p-1} \Delta s}{\int_{a}^{b}(g(s)+h(s))^{p} \Delta s}\right|^{p} \Delta t\right)^{\frac{1}{p}} \\
& +\left(\int_{a}^{b} h^{p}(t) \Delta t-\int_{a}^{b}\left|h(t)-(g(t)+h(t)) \frac{\int_{a}^{b} h(s)(g(s)+h(s))^{p-1} \Delta s}{\int_{a}^{b}(g(s)+h(s))^{p} \Delta s}\right|^{p} \Delta t\right)^{\frac{1}{p}}
\end{aligned}
$$

is valid.

Proof. The inequality (10) follows directly from Theorem 4.1 and Theorem 2.6 .

Remark 4.3. If the functions $g$ and $h$ in Theorem 4.2 are nonnegative, then inequality (10) represents a refinement of Minkowski's inequality on time scales as established in [5, Theorem 3.3] since the delta integral is an isotonic linear functional.

\section{Jessen-Mercer's inequality}

A variant of Jensen's inequality of Mercer's type for superquadratic functions and isotonic linear functionals is given in the following theorem.

Theorem 5.1 (See $\left[2\right.$, Theorem 2.3]). Let L satisfy properties $\left(\mathrm{L}_{1}\right),\left(\mathrm{L}_{2}\right)$, on a nonempty set $E$, let $\varphi:[0, \infty) \rightarrow \mathbb{R}$ be a continuous superquadratic function, and let $0 \leq m<M<\infty$. Assume that $A$ is an isotonic linear functional on $L$ with $A(1)=1$. If $g \in L$ is such that $m \leq g(t) \leq M$ for all $t \in E$ and such that $\varphi(g), \varphi(m+M-g),(M-g) \varphi(g-m),(g-m) \varphi(M-g), \varphi(|g-A(g)|) \in L$, then we have

$$
\begin{aligned}
\varphi(m+M-A(g)) \leq & \varphi(m)+\varphi(M)-A(\varphi(g)) \\
& -\frac{2}{M-m} A((g-m) \varphi(M-g)+(M-g) \varphi(g-m)) \\
& -A(\varphi(|g-A(g)|)) .
\end{aligned}
$$


If the function $\varphi$ is subquadratic, then the above inequality is reversed.

Next, we state the time scales version of Jensen's inequality of Mercer's type for superquadratic functions and isotonic linear functionals which we will call the Jessen-Mercer inequality for superquadratic functions on time scales.

Theorem 5.2. Let $a, b \in \mathbb{T}$. Let $\varphi:[0, \infty) \rightarrow \mathbb{R}$ be a continuous superquadratic function and $0 \leq m<M<\infty$. If $g:[a, b]_{\mathbb{T}} \rightarrow[m, M]$ is an rd-continuous function, then

$$
\begin{aligned}
& (b-a) \varphi\left(m+M-\frac{1}{b-a} \int_{a}^{b} g(t) \Delta t\right) \\
\leq & (\varphi(m)+\varphi(M))(b-a)-\int_{a}^{b} \varphi(g(t)) \Delta t \\
& -\frac{2}{M-m} \int_{a}^{b}[(g(t)-m) \varphi(M-g(t))+(M-g(t)) \varphi(g(t)-m)] \Delta t \\
& -\int_{a}^{b}\left[\varphi\left(\left|g(t)-\frac{1}{b-a} \int_{a}^{b} g(s) \Delta s\right|\right)\right] \Delta t .
\end{aligned}
$$

Proof. The result follows from Theorem 2.6, with $A$ there replaced by

$$
A(f)=\frac{1}{b-a} \int_{a}^{b} f(t) \Delta t
$$

and Theorem 5.1 .

\section{Converse of Jensen's inequality}

In the following theorem, a functional version of the converse of Jensen's inequality for superquadratic functions is recalled.

Theorem 6.1 (See [8, Theorem 15]). Let L satisfy conditions $\left(\mathrm{L}_{1}\right),\left(\mathrm{L}_{2}\right)$ and $A$ satisfy conditions $\left(\mathrm{A}_{1}\right)$ and $\left(\mathrm{A}_{2}\right)$ on a nonempty set $E$. Let $k \in L$ be a nonnegative function. Suppose that $\varphi:[0, \infty) \rightarrow \mathbb{R}$ is a superquadratic function. Then for every $f \in L, f: E \rightarrow[m, M] \subseteq[0, \infty)$ such that $k f, k(\varphi \circ f) \in L$, we have

$$
A(k \varphi(f))+\Delta_{c} \leq \frac{M A(k)-A(k f)}{M-m} \varphi(m)+\frac{A(k f)-m A(k)}{M-m} \varphi(M),
$$

where

$$
\Delta_{c}=\frac{1}{M-m} A((M k-k f) \varphi(f-m \cdot 1)+(k f-m k) \varphi(M \cdot 1-f)) .
$$

Now, we give a converse of Jensen's inequality for superquadratic functions on time scales. 
Theorem 6.2. Suppose $a, b \in \mathbb{T}$ and $\varphi:[0, \infty) \rightarrow \mathbb{R}$ is a superquadratic function. Then for every $f:[a, b]_{\mathbb{T}}^{\kappa} \rightarrow[m, M] \subseteq[0, \infty)$ such that $f, \varphi \circ f \in \mathrm{C}_{\mathrm{rd}}$, we have

$\int_{a}^{b} \varphi(f(t)) \Delta t+R \leq \frac{M(b-a)-\int_{a}^{b} f(s) \Delta s}{M-m} \varphi(m)+\frac{\int_{a}^{b} f(s) \Delta s-m(b-a)}{M-m} \varphi(M)$,

where

$$
R=\frac{1}{M-m} \int_{a}^{b}[(M-f(t)) \varphi(f(t)-m)+(f(t)-m) \varphi(M-f(t))] \Delta t .
$$

Proof. Inequality (11) follows directly from Theorem 2.6 and Theorem 6.1 with $k(t)=1$ for all $t \in[a, b]_{\mathbb{T}}^{\kappa}$.

\section{Slater's inequality}

A functional Slater type inequality for superquadratic functions, which gives another estimate of the expression $A(\varphi(f))$, is given next.

Theorem 7.1 (See [8, Theorem 17]). Let L satisfy conditions $\left(\mathrm{L}_{1}\right),\left(\mathrm{L}_{2}\right)$ and $A$ satisfy conditions $\left(\mathrm{A}_{1}\right)$ and $\left(\mathrm{A}_{2}\right)$ on a nonempty set $E$. Suppose that $\varphi$ : $[0, \infty) \rightarrow \mathbb{R}$ is a superquadratic function, $C$ is as in Definition 1.6, and $k, f \in L$ are nonnegative functions such that $k \varphi(f), k C(f), k f C(f), k \varphi(|f-M \cdot 1|) \in$ L. If

$$
M=\frac{A(k f C(f))}{A(k C(f))} \geq 0
$$

then

$$
A(k \varphi(f)) \leq \varphi(M) A(k)-A(k \varphi(|f-M \cdot 1|)) .
$$

Now, we can state the Slater type inequality for superquadratic functions on time scales.

Theorem 7.2. Suppose $a, b \in \mathbb{T}, \varphi:[0, \infty) \rightarrow \mathbb{R}$ is a superquadratic function, and $C$ is as in Definition 1.6. Let $f:[a, b]_{\mathbb{T}}^{\kappa} \rightarrow[0, \infty)$ and $f, \varphi, C \in \mathrm{C}_{\mathrm{rd}}$. If $C$ is a nonnegative function, then

$$
\int_{a}^{b} \varphi(f(t)) \Delta t \leq \varphi(M)(b-a)-\int_{a}^{b} \varphi(|f(t)-M|) \Delta t
$$

where

$$
M=\frac{\int_{a}^{b} f(t) C(f(t)) \Delta t}{\int_{a}^{b} C(f(t)) \Delta t} .
$$

Proof. Inequality (12) follows directly from Theorem 2.6 and Theorem 7.1 with $k(t)=1$ for all $t \in[a, b]_{\mathbb{T}}^{\kappa}$. 


\section{Hermite-Hadamard's inequality}

In this section, using the properties of superquadratic functions, we refine the Hermite-Hadamard inequality for time scales as given in [11, Theorem 3.9].

For proving the Hermite-Hadamard inequality on time scales, a delta integral of the form $\int_{a}^{b} t \Delta t$ must be evaluated. For an arbitrary time scale, it is known that an antiderivative of 0 is 1 , an antiderivative of 1 is $t$, but it is not possible to find a closed formula of an antiderivative of $t$. More details on that subject can be found in [10, Section 1.6].

Using the basics of the time scale calculus and the combined dynamic derivative (so-called $\nabla_{\alpha}$ derivative), in [11] it is shown that for all time scales $\mathbb{T}$ and all $\alpha \in[0,1]$, we have

$$
x_{\alpha}:=\frac{1}{b-a} \int_{a}^{b} t \diamond_{\alpha} t \in[a, b] .
$$

Also, the Hermite-Hadamard inequality for time scales is given as follows.

Theorem 8.1 (See [11, Theorem 3.9]). Let $\mathbb{T}$ be a time scale and $a, b \in \mathbb{T}$. Let $f:[a, b] \rightarrow \mathbb{R}$ be a continuous convex function. Then

$$
f\left(x_{\alpha}\right) \leq \frac{1}{b-a} \int_{a}^{b} f(t) \diamond_{\alpha} t \leq \frac{b-x_{\alpha}}{b-a} f(a)+\frac{x_{\alpha}-a}{b-a} f(b) .
$$

As the diamond- $\alpha$ derivative reduces to the $\Delta$-derivative for $\alpha=1$, using the above results from [11] and the results given in Theorem 2.5 and Theorem 6.2 , we can now state the Hermite-Hadamard inequality for superquadratic functions on time scales.

Theorem 8.2. Let $\mathbb{T}$ be a time scale and $a, b \in \mathbb{T}$. Suppose $\varphi:[0, \infty) \rightarrow \mathbb{R}$ is a continuous superquadratic function. Then

$$
\begin{aligned}
& \varphi(\bar{x})+\frac{1}{b-a} \int_{a}^{b} \varphi(|t-\bar{x}|) \Delta t \\
\leq & \frac{1}{b-a} \int_{a}^{b} \varphi(t) \Delta t \\
\leq & \frac{b \varphi(a)-a \varphi(b)}{b-a}+\frac{\varphi(b)-\varphi(a)}{b-a} \bar{x} \\
& -\frac{1}{(b-a)^{2}} \int_{a}^{b}[(b-t) \varphi(t-a)+(t-a) \varphi(b-t)] \Delta t,
\end{aligned}
$$

where

$$
\bar{x}=\frac{1}{b-a} \int_{a}^{b} t \Delta t .
$$

Proof. The left-hand side of (14) follows from the refinement of Jensen's inequality on time scales given in inequality (3) of Theorem 2.5, taking $f(t)=t$ for all $t \in[a, b]_{\mathbb{T}}^{\kappa}$, and using (13) for $\alpha=1$. To prove the right-hand side of 
(14), it suffices to put $m=a, M=b$, and $f(t)=t$ for all $t \in[a, b]_{\mathbb{T}}^{\kappa}$ in the converse of Jensen's inequality for superquadratic functions on time scales as given in inequality (11) of Theorem 6.2.

Remark 8.3. The same result, i.e., inequality (14), can be obtained using the considerations demonstrated in the previous sections. Namely, joining the inequalities established in Theorem 2.3 and Theorem 6.1, the Hermite-Hadamard type inequality can be written out for superquadratic functions and isotonic linear functionals. Taking in such an inequality $f(t)=t, m=a, M=b, k=1$ and replacing the functional $A$ with the delta integral from $a$ to $b$ gives us exactly inequality (14).

Remark 8.4. In the case when $\varphi$ is a nonnegative superquadratic function and therefore (by Lemma 1.7) a convex one too, the result of Theorem 8.2 refines the result given in Theorem 8.1 because, according to Theorem 2.6, the delta integral satisfies property $\left(\mathrm{A}_{2}\right)$ of being an isotonic functional.

\section{References}

[1] S. Abramovich, S. Banić, M. Matić, and J. Pečarić, Jensen-Steffensen's and related inequalities for superquadratic functions, Math. Inequal. Appl. 11 (2008), no. 1, 23-41.

[2] S. Abramovich, J. Barić, and J. Pečarić, A variant of Jessen's inequality of Mercer's type for superquadratic functions, J. Inequal. Pure Appl. Math. 9 (2008), no. 3, Article 62, 13 pages.

[3] S. Abramovich, G. Jameson, and G. Sinnamon, Inequalities for averages of convex and superquadratic functions, J. Inequal. Pure Appl. Math. 5 (2004), no. 4, Article 91, 14 pp.

[4] , Refining Jensen's inequality, Bull. Math. Soc. Sci. Math. Roumanie (N.S.) 47(95) (2004), no. 1-2, 3-14.

[5] R. P. Agarwal, M. Bohner, and A. Peterson, Inequalities on time scales: a survey, Math. Inequal. Appl. 4 (2001), no. 4, 535-557.

[6] M. Anwar, R. Bibi, M. Bohner, and J. Pečarić, Integral inequalities on time scales via the theory of isotonic linear functionals, Abstr. Appl. Anal. 2011 (2011), Art. ID 483595, 16 pp.

[7] S. Banić, J. Pečarić, and, S. Varošanec, Superquadratic functions and refinements of some classical inequalities, J. Korean Math. Soc. 45 (2008), no. 2, 513-525.

[8] S. Banić and S. Varošanec, Functional inequalities for superquadratic functions, Int. J. Pure Appl. Math. 43 (2008), no. 4, 537-549.

[9] J. Barić, Superquadratic functions, PhD thesis, University of Zagreb, Zagreb, Croatia, 2007.

[10] M. Bohner and A. Peterson, Dynamic Equations on Time Scales, Birkhäuser Boston Inc., Boston, MA, 2001.

[11] C. Dinu, Hermite-Hadamard inequality on time scales, J. Inequal. Appl. 2008 (2008), Art. ID 287947, 24 pp.

[12] S. Hilger, Ein Maßkettenkalkül mit Anwendung auf Zentrumsmannigfaltigkeiten, PhD thesis, Universität Würzburg, 1988.

[13] - Analysis on measure chains-a unified approach to continuous and discrete calculus, Results Math. 18 (1990), no. 1-2, 18-56.

[14] _ Differential and difference calculus-unified!, Proceedings of the Second World Congress of Nonlinear Analysts, Part 5 (Athens, 1996). Nonlinear Anal. 30 (1997), no. 5, 2683-2694. 
TIME SCALES INTEGRAL INEQUALITIES FOR SUPERQUADRATIC FUNCTIONS 477

[15] J. L. W. V. Jensen, Sur les fonctions convexes et les inégalités entre les valeurs moyennes, Acta Math. 30 (1906), no. 1, 175-193.

[16] J. Pečarić, F. Proschan, and Y. L. Tong, Convex Functions, Partial Orderings, and Statistical Applications, volume 187 of Mathematics in Science and Engineering, Academic Press Inc., Boston, MA, 1992.

\section{Josipa BARIĆ}

FACUlty of Electrical Engineering

Mechanical Engineering and Naval Architecture

UNIVERSITY OF SPLIT

RuĐera BošKovića 32, 21000 Split, Croatia

E-mail address: jbaric@fesb.hr

RABIA BIBI

Centre for Advanced Mathematics and Physics

National University of Sciences and Technology

Islamabad, PAKISTAN

E-mail address: emaorr@gmail.com

Martin Bohner

Department of Mathematics and Statistics

Missouri University of Science and Technology

Rolla, MisSOURI 65409-0020, USA

E-mail address: bohner@mst.edu

Josip PEČARIĆ

Faculty of Textile Technology

UNIVERSITY OF ZAGREB

Pierottijeva 6, 10000 Zagreb, Croatia

E-mail address: pecaric@mahazu.hazu.hr 